\title{
CHANGE DETECTION OF MULTI-POLARIMETRIC SAR DATA BASED ON PRINCIPAL COMPONENT ANALYSIS
}

\author{
X. Chen, T. Wu, N. Niu \\ $38^{\text {th }}$ Research Institute, China Electronic Technology Corporation, Hefei, China \\ xichen@126.com
}

KEY WORDS: Polarimetric SAR, Change detection, Principal component analysis

\begin{abstract}
:
Recently, Polarimetric SAR (PolSAR) techniques have been much studied as hot research topics in the area of SAR. The objective of this paper is to assess the Principal component analysis (PCA) technique combining with multi-polarimetric SAR data for change detection. PCA proposed in this paper give an effective and quick way to achieve the difference map from the whole multi-temporal images, and while the multi-polarimetric SAR present more information than traditional single-polarimetric SAR and can give an accurate result for change detection.

Finally, the simulated multi-temporal SAR images from ERS are used to validate the PCA technique, and then ENVISAT dual-pol SAR data and ALOS PALSAR full-pol SAR data are applied to present the preliminary results of change detection. The results show that the polarimetric PCA technique proposed here has a good performance on those experimental SAR data, because it take full advantage of two components corresponding to unchanged and changed portion and the polarimetric information.
\end{abstract}

\section{INTRODUCTION}

Change detection is an important technique in environmental earth observation and security, and implies the comparison of remote sensing images from different moments in time. Despite the presence of speckle noise, the use of SAR sensors in change detection is potentially attractive from the operational viewpoint. The active microwave sensors present the advantage that (unlike optical ones) they are independent of atmospheric and sunlight conditions. This makes it possible to plan the monitoring of a region (by repeat pass imaging) with advance timing defined according to end-user requirements (e.g., seasonal and agricultural calendars). In the context of SAR image analysis, the problem of unsupervised change detection has been addressed focusing on different aspects, including image despeckling, choice of the comparison operator and optimal threshold selection. Because of the multiplicative nature of speckle noise, it appears more effective to use the ratio operator than the difference operator to compare two SAR temporal images. In fact, the ratio method is quite sensitive to the presence of image speckle in the sense that speckle patterns[1].

Principle component analysis(PCA) is usually adoped for compression and enhancement of multispectral data. More recently PCA has applied on multifrequency polarimetric SAR imagery[2] by considering a multiplicative model to describe the interaction between signal and speckle noise with effective results.

After PC transformation, the set of the transformed data presents several favorable properties. Images are uncorrelated with each other. The variance of the data is equal in both domains and is strongly concentrated in the first component. The signal to noise ratio(SNR) of the first component increases with respect to the SNR of each original image, resulting in a visual enhancement with associated reduction of noise. Common features of the images appear in the first component while differences in the others. This work deals with the application of PCA to multitemporal polarimetric SAR data[3,4], aiming at detecting changes in the backscatter response.

\section{PCA TECNIQUE}

\subsection{General Theory}

Two SAR image vectors $X_{1} 、 X_{2}$ with the same region and different time are projected to the principal direction $V_{P}$ and second direction $V_{M}$ [5],

$$
\begin{aligned}
& X_{1}=X_{1 P}+X_{1 M} \\
& X_{2}=X_{2 P}+X_{2 M}
\end{aligned}
$$

Where,

$$
\begin{aligned}
& X_{1 P}, X_{2 P}=\text { same parts of two SAR images } \\
& X_{1 M}, X_{2 M}=\text { different parts of two SAR images }
\end{aligned}
$$

Set $A=\left[X_{1}, X_{2}\right]$, from SVD's(Singular Values decomposition) view, we can get

where

$$
A=V D U^{T}
$$

$$
\begin{aligned}
& V=\left[v_{1}, v_{2}, \ldots, v_{m}\right] \\
& D=\left[\begin{array}{c}
D_{1} \\
0
\end{array}\right] \\
& D_{1}=\operatorname{diag}\left(\lambda_{1}, \lambda_{2}\right) \\
& U=\left[u_{1}, u_{2}\right]
\end{aligned}
$$

Because of the orthogonality of vector $\mathrm{V}$ and vector $\mathrm{U}$, we can also get

$$
\begin{gathered}
A=\lambda_{1} v_{1} u_{1}^{T}+\lambda_{2} v_{2} u_{2}^{T} \\
B=X_{1} X_{1}^{T}+X_{2} X_{2}^{T}=A A^{T}=V D D^{T} V^{T}
\end{gathered}
$$

And then,

$$
\begin{aligned}
& X_{1 p}=\left\|X_{1}\right\| \frac{\left\langle X_{1}, v_{1}\right\rangle}{\left\|X_{1} \mid\right\| v_{1} \|} v_{1}=v_{1} v_{1}^{T} X_{1} \\
& X_{2 p}=\left\|X_{2}\right\| \frac{\left\langle X_{2}, v_{1}\right\rangle}{\left\|X_{2} \mid\right\| v_{1} \|} v_{1}=v_{1} v_{1}^{T} X_{2}
\end{aligned}
$$

Thus,

$$
\left[X_{1 P}, X_{2 P}\right]=v_{1} v_{1}^{T} A
$$


According to equation(2)and equation(8), we can get Similarly,

$$
\left[X_{1 P}, X_{2 P}\right]=v_{1} v_{1}^{T}\left(\lambda_{1} v_{1} u_{1}^{T}+\lambda_{2} v_{2} u_{2}^{T}\right)=\lambda_{1} v_{1} u_{1}^{T}
$$

$$
\left[X_{1 M}, X_{2 M}\right]=\lambda_{2} v_{2} u_{2}^{T}
$$

\subsection{Combining with Polarimetric SAR Information}

For the sake of reduce of computation cost, we use $G=A^{T} A$, whose size is $2 * 2$. And then, matrix $G$ can be written as

$$
\begin{aligned}
G & =A^{T} A=U D^{T} D U^{T} \\
A u_{1} u_{1}^{T} & =\left(\lambda_{1} v_{1} u_{1}^{T}+\lambda_{2} v_{2} u_{2}^{T}\right) u_{1} u_{1}^{T}=\lambda_{1} v_{1} u_{1}^{T} \\
A u_{2} u_{2}^{T} & =\left(\lambda_{1} v_{1} u_{1}^{T}+\lambda_{2} v_{2} u_{2}^{T}\right) u_{2} u_{2}^{T}=\lambda_{2} v_{2} u_{2}^{T}
\end{aligned}
$$

thus

$$
\begin{aligned}
& {\left[X_{1 P}, X_{2 P}\right]=A u_{1} u_{1}^{T}} \\
& {\left[X_{1 M}, X_{2 M}\right]=A u_{2} u_{2}^{T}}
\end{aligned}
$$

In this paper, PCA technique with polarimetric SAR data will be done as follows:

Step1: speckle filtering for multi-temporal polSAR data;

Step2: change detection based on ratio method for different polarization data, such as $\mathrm{HH}, \mathrm{VV}$;

Step3: Using the matrix G and applying PCA analysis to two results of change detection for different polarization data; Step4: setting a threshold $\mathrm{T}$ for change detection, and getting the final change detection result from the principle component.

\section{EXPERIMENTAL RESULTS}

\subsection{Test for Simulated SAR data}

The PCA technique proposed in this paper will be firstly validated using a simulated multi-temporal SAR data which came from ERS SAR data imaged on 11 January 2004. The dark rectangle as changed part in simulated data was made by hand.

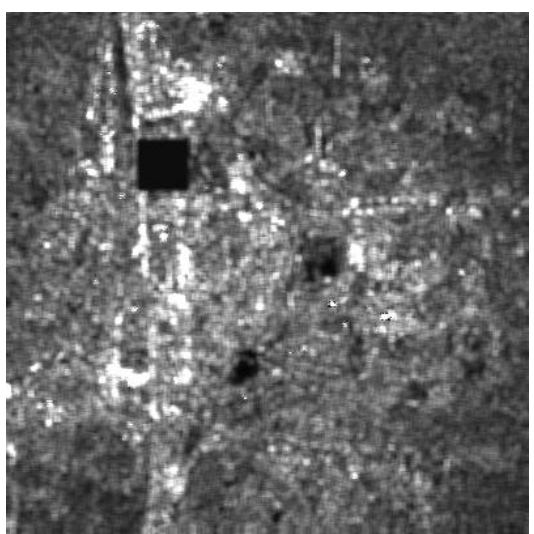

Figure 1. Simulated SAR data with changed part

For the single-polarization SAR data, change detection based on PCA technique need get the second vector $\left[X_{1 M}, X_{2 M}\right]$, and then $\Delta X=X_{1 M}-X_{2 M}$ is the changed part between two temporal SAR data. In Figure2, it obvious that PCA technique achieved a good result as expected. But it is just a simple simulated sample, and the test for real polarimetric data is necessary.

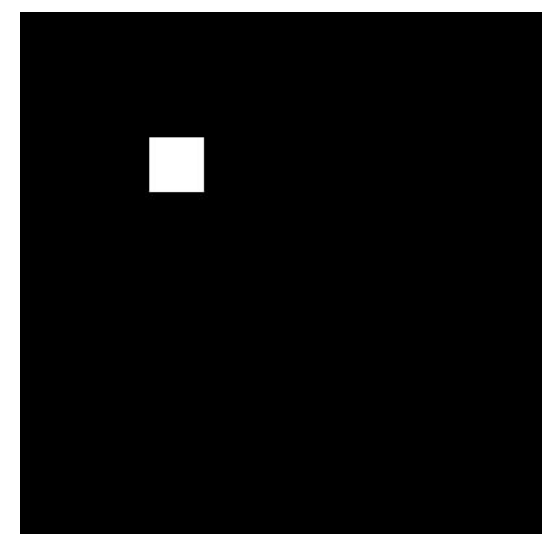

Figure 2. Change detection result using PCA technique

\subsection{Test for Real Polarimetric SAR data}

ENVISAT dual-polarization(HH and VV) SAR data imaged on 2004 and 2005 respectively in Figure3 and Figure 4 are used for change detection.

According to the four steps proposed in section2, the principal part are extracted from $\mathrm{HH}$ and VV difference maps using ratio method. Figure5 presents the difference map after PCA analysis, and the final change detection result is shown in Figure6.

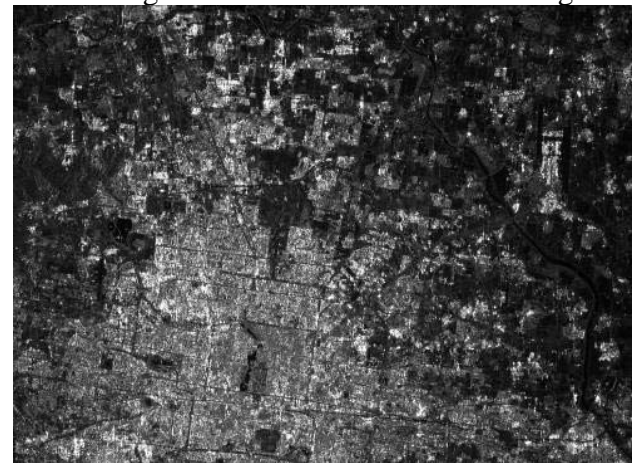

Figure 3. ENVISAT SAR data(HH, 2004)

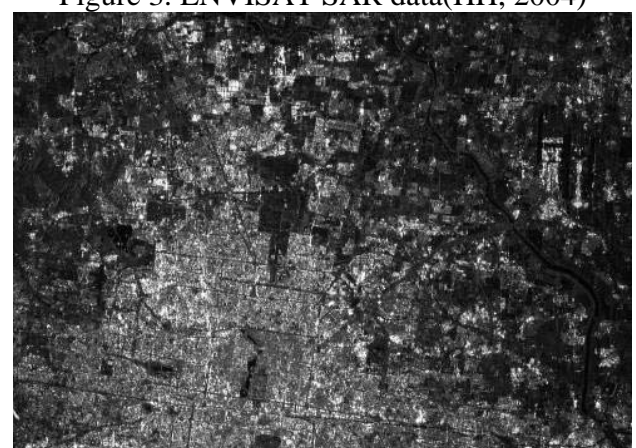

Figure 4. ENVISAT SAR data(HH, 2005)

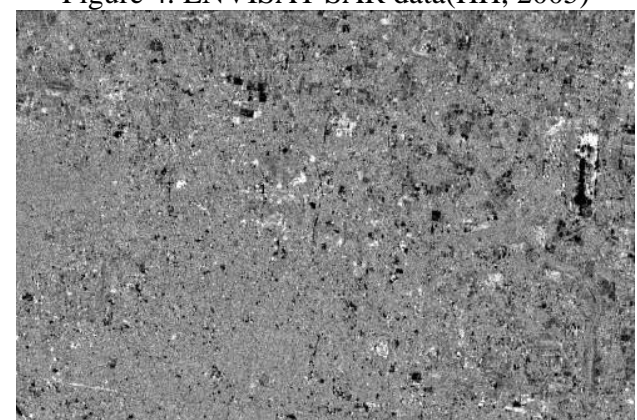

Figure 5. Difference map after PCA analysic 


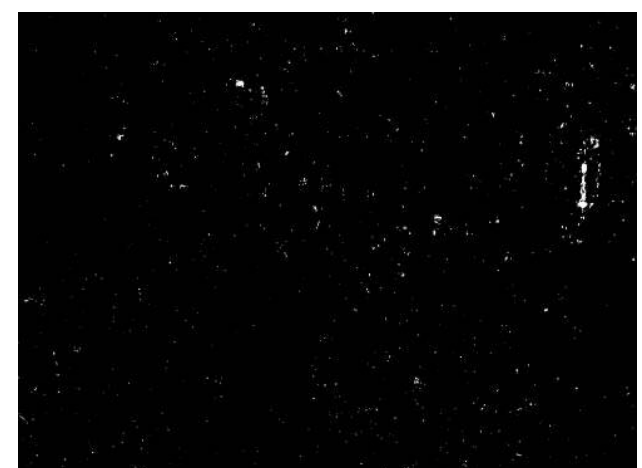

Figure 6. Final change detection result Moreover, PALSAR full-polarization SAR data imaged on 2005 and 2006 respectively in Figure7 are used for change detection. Similarly, Figure8 presents the difference map after PCA analysis and the final change detection resul.

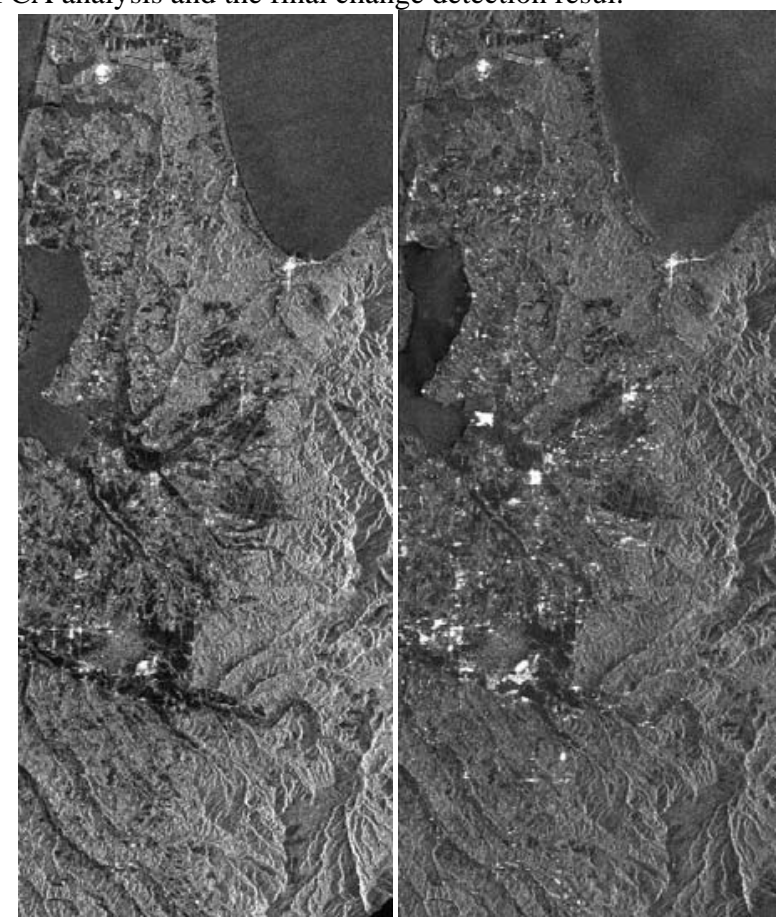

Figure 7. PALSAR multi-temporal SAR data(HH, 2005-left and 2006-right)

\section{CONCLUSION}

In this paper, we proposed a simple method for change detection combining with the PCA transform and polarimetric SAR information in the final results, which need less computation cost for Remote Sensing application. All the tests on the simulated and real SAR data show that this is an effective process and achieved the result as expected. However, the investigation in the role of full polarimetric SAR information in the result of multi-temporal change detection requires study in the Further works.

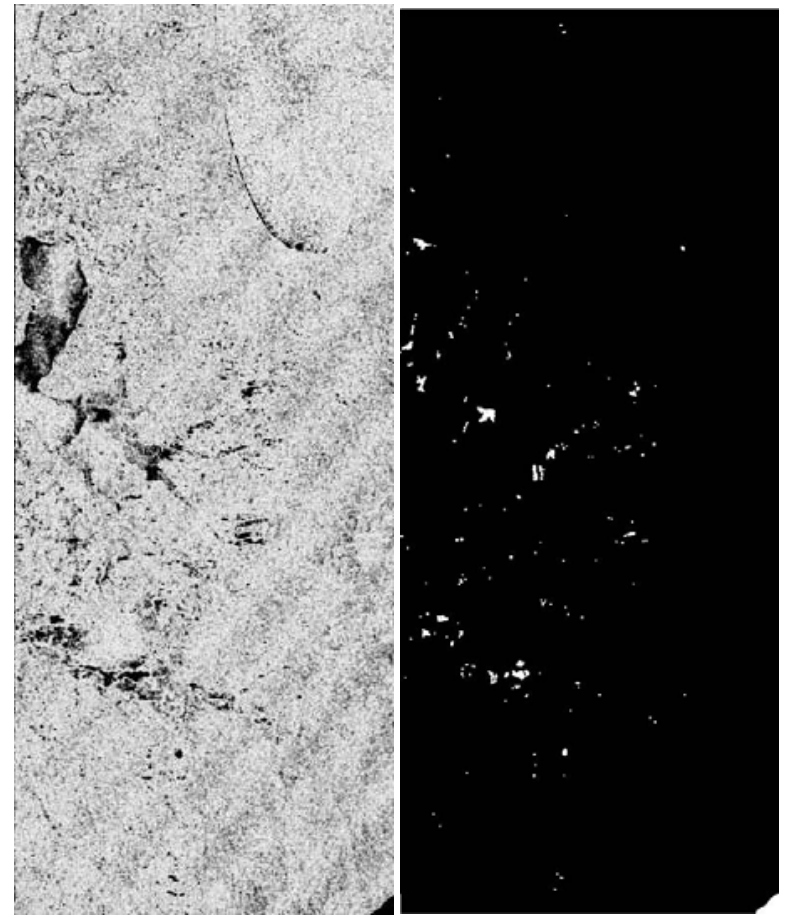

Figure 8. difference map after PCA analysis and final change detection result

\section{REFERENCES}

1.Singh, A. 1989. Review Article: Digital change detection techniques using remotely-sensed data.Int. J. Remote Sensing, vol.10, no.6, pp.989-1003.

2.Lee J.S. and Hoppel K. 1992. Principal Compents Transformation of Multifrequency Polarimetric SAR Imagery. IEEE Trans. Geosci. Remote Sensing, vol.30, no.4, pp.686-696. 2.W. Dierking, H. Skriver.2002.Change detection for thematic mapping by means of airborne multitemporal polarimetric SAR Imagery. IEEE Trans. Geosci. Remote Sens., vol. 40, no. 3, pp. 618- 636.

3.Qiu B, Prinet V, Perrier E, and Monga O. 2003. Multi-block PCA method for image change detection. Proceedings of the 12th International Conference on Image Analysis and Processing, Mantova, Italy, 17-19 Sept, pp.385-390.

5.Zhang Hui, Wang Jian-guo. 2008. A SAR image Change Detection Algorithm Based on Principal Component Analysis. Journal of Electronics \& Information Technology, vol.30, no.7, 1727-1730.

\section{ACKNOWLEDGMENT}

This research is supported by the National High Technology Research and Development Program of China(863 Program)(No.2011AA120404). 